\title{
Efficacy of miltefosine compared with glucantime for the treatment of cutaneous leishmaniasis: a systematic review and meta-analysis
}

\author{
Sohrab Iranpour ${ }^{1,2}$, Ali Hosseinzadeh ${ }^{3}$, Abbas Alipour ${ }^{4}$ \\ 'Student Research Committee, Department of Epidemiology, Faculty of Public Health and Safety, Shahid Beheshti University of Medical \\ Sciences, Tehran, Iran; ${ }^{2}$ Department of Community Medicine, Faculty of Medicine, Ardabil University of Medical Sciences, Ardabil, Iran; ${ }^{3}$ Research \\ Center for Modeling in Health, Institute for Future Studies in Health, Kerman University of Medical Sciences, Kerman, Iran; ${ }^{4}$ Department of \\ Community Medicine, Faculty of Medicine, Mazandaran University of Medical Sciences, Mazandaran, Iran
}

\begin{abstract}
Cutaneous leishmaniasis (CL) is most common form of leishmaniasis and is characterized by ulcerative skin lesions. The objective of this study was to conduct a systematic review and meta-analysis of clinical trials that compared the efficacy of miltefosine and glucantime for the treatment of CL. We searched the following databases: Cochrane, PubMed, Embase, Scopus, Web of Science, ProQuest, Cochrane Central Register of Controlled Trials, International Clinical Trials Registry Platform search portal of World Health Organization, Sid, Irandoc, Magiran, and clinicaltrials.gov. We used keywords including "miltefosine," "glucantime," and "Leishmania." The quality of studies was assessed using the Cochrane risk of bias tool. A random-effects model was employed for the analysis. We assessed heterogeneity by the chi-square test and the $\mathrm{I}^{2}$ index statistic. When heterogeneity was present, meta-regression analyses were performed. The Egger method was used to assess publication bias; when it was significant, the trim-and-fill method was used to test and adjust for publication bias. A total of 1,570 reports were identified, of which 10 studies were included in the meta-analysis. In the meta-analysis, there was no significant difference between the efficacy of miltefosine and glucantime; however, subgroup analysis showed that, regarding parasite species other than Leishmania braziliensis, miltefosine was significantly superior to glucantime (intention to treat; relative risk, 1.15; 95\% confidence interval, 1.01 to 1.32 ). In the meta-regression, only the glucantime injection type was significant at the $\mathrm{p}=0.1$ level. The Egger test found statistically significant publication bias; however, including the 3 missing studies in the trim-and-fill analysis did not change the results. This meta-analysis found that miltefosine seems to be more effective than glucantime, at least in species other than L. braziliensis, for treating CL.
\end{abstract}

KEY WORDS: Efficacy, Miltefosine, Glucantime, Cutaneous leishmaniasis, Systematic review, Meta-analyses

\section{INTRODUCTION}

Leishmaniasis is a protozoan parasitic infection that, depending on the infecting species of Leishmania, can manifest in different

\section{Correspondence: Abbas Alipour}

Department of Community Medicine, Faculty of Medicine, Mazandaran University of Medical Sciences, Mazandaran 198353-5511, Iran Email: alipour.abbas59@gmail.com

Received: Mar 1, 2019 / Accepted: Mar 31, 2019 / Published: Mar 31, 2019

This article is available from: http://e-epih.org/

(C) This is an open-access article distributed under the terms of the Creative Commons Attribution License (http://creativecommons.org/licenses/by/4.0/), which permits unrestricted use, distribution, and reproduction in any medium, provided the original work is properly cited.

(C) 2019, Korean Society of Epidemiology forms, including cutaneous, mucocutaneous, or visceral leishmaniasis [1]. Infections occur all over the world; however, it is endemic in 98 countries, predominantly tropical and subtropical. Over 350 million people are at risk globally, with 1.3 million new cases each year and 20,000 to 40,000 deaths annually [2,3]. Cutaneous leishmaniasis (CL) can be caused by a variety of species, including Leishmania braziliensis, Leishmania amazonensis, Leishmania aethiopica, Leishmania mexicana, Leishmania guyanensis, Leishmania panamensis, Leishmania peruviana, Leishmania tropica and Leishmania major. CL, characterized by ulcerative skin lesions, is the least fatal but most common form of the disease [1]. CL is widely distributed; however, $70 \%$ to $75 \%$ of the estimated global incidence is accounted for by 10 countries, including Brazil, Colombia, North Sudan, Iran, Afghanistan, Algeria, Syria, Ethiopia, Costa Rica, and Peru [2]. In such countries, although of the local 
population seem to be developing some immunity [4], others such as travelers, tourists, workers, and military troops are more at risk and may also inadvertently carry the disease to non-endemic areas [5,6]. Although CL is a self-limited disease, in some cases it may be severe and lead to mucosal disease or disseminated leishmaniasis [7-9]. Since the 1940s, pentavalent antimonials (meglumine antimoniate [MA] and sodium stibogluconate) have been considered to be the first line of treatment for leishmaniasis [10]; indeed, they are widely used for the treatment of all forms of leishmaniasis [11]. Some of the well-known problems associated with using pentavalent antimonials include the frequency and severity of adverse symptoms and difficulties in their administration [11]. With regard to the former factors, these drugs are very toxic and may affect organs such as the pancreas, liver, kidney, and the hematological system [12]. Some serious side effects include cardiac arrhythmia, pancreatitis, myalgia, arthralgia, liver enzyme elevation, the possible need for repeated parenteral injections [1316], fever, and headache [17]. Therefore, it is clear that the use of pentavalent antimonials could pose life-threatening risks. Additionally, drug resistance has become widespread [13]; in previous studies, a decrease in the efficacy of MA and a decrease in sensitivity of Leishmania parasites to antimonials have been reported [10,18-21]. An increasing number of Iranian patients are unresponsive to MA as a first-line treatment for leishmaniasis; indeed, it has been demonstrated that in about $40 \%$ of CL cases, there is no desirable beneficial response to MA during the first course of treatment $[22,23]$. Furthermore, in certain regions where antimony compounds have been used for a long time, $40 \%$ of cases show disadvantages in terms of drug toxicity and subsequent clinical resistance $[15,24,25]$. The decreasing sensitivity of Leishmania parasites to MA and the potential irregular adherence to the daily schedule of the parenteral route during the first 20 days are the main factors underlying the decreasingly desirable response rate to MA [26,27]. Therefore, to better control CL, it is necessary to develop new therapeutic strategies with a higher efficacy and safety rate, coupled with better patient adherence [28]. This would require a greater push towards more productive research into therapeutic alternatives for the treatment of leishmaniasis [17]. Miltefosine, a phosphatidylcholine analogue, is an antileishmanial oral drug that has been shown to be $>95 \%$ curative for visceral leishmaniasis in India [29]. A high cure rate of CL (91\%) has also been demonstrated after oral treatment with miltefosine [30,31]. In one study, miltefosine cured $88 \%$ of patients with aggressive L. major infections that did not respond to intralesional antimony [32]. Miltefosine is a safe and effective oral treatment for CL [33-35] that could be used as an option for CL therapy instead of MA. However, findings from studies that have compared MA and miltefosine are inconsistent. For instance, in some studies, the therapeutic efficacy of oral miltefosine was not significantly different from that of MA $[11,33,36-38]$. In other studies, the efficacy of miltefosine was statistically significantly superior to that of MA in the treatment of CL $[28,35,39]$. However, another randomized clinical trial (RCT) found a significant difference favoring MA
[12]. In a systematic review and meta-analysis published in 2013 that included 5 studies conducted in American countries, no significant difference was found between miltefosine and MA in the complete cure rate at 6 months. However, when pooling 2 studies focused on L. panamensis and L. guyanensis species, a significant difference favoring miltefosine was found in the rate of complete cure at 6 months [40]. The objective of this paper is to present a systematic review and meta-analysis of clinical trials that compared the efficacy of miltefosine and MA in the treatment of CL in countries throughout the world.

\section{MATERIALS AND METHODS}

In this study, we adhered to the guidelines of the 2009 PRISMA (Preferred Reporting Items for System reviews and Meta-Analyses) statement for reporting systematic reviews and meta-analyses of RCTs.

\section{Literature search and data sources}

Literature searches of electronic databases from 1991 to July 31, 2017, were conducted in August 2017. We searched the Cochrane Library, PubMed, Embase, Scopus, Web of Science, ProQuest, Cochrane Central Register of Controlled Trials, International Clinical Trials Registry Platform search portal of WHO, 3 Persian-language databases (Sid, Irandoc, and Magiran), and clinicaltrials. gov. In addition, hand-searching of the references of the included articles and previous reviews and meta-analyses constituted secondary search strategy to find other eligible trials. Gray literature papers, book chapters, and the main journals in the field of leishmaniasis were also searched. There were no language restrictions in the searches. All retrieved references were managed with a reference manager program (EndNote).

This search used the following keywords: "miltefosine," "glucantime," and "Leishmania".

The PubMed systematic search strategy is presented in Supplementary Material 1 . This search strategy was amended with adjustments in vocabulary and syntax for each database.

\section{Interventions and comparisons}

The interventions were miltefosine and MA through any route of administration. The primary efficacy outcome of interest was the complete cure rate at 6 months.

\section{Systematic review process}

For screening, titles and abstracts from the primary search were independently assessed by 2 reviewers (SI and $\mathrm{AH}$ ) for the full text of the studies according to the selection criteria. For further screening, the full text of all potentially eligible studies was then assessed using the inclusion and exclusion criteria by the same 2 reviewers. Disagreements were solved by discussion and consensus.

The original search included all study designs, but only clinical trials were included in the analyses for this paper. Studies were included if they involved patients of any age of male and/or female 
sex, reported efficacy in human participants, and contained enough information to extract data for an intention-to-treat (ITT) analysis. Articles were excluded if they described animal, in vitro, or in vivo experimental studies or if they were expert opinion papers.

\section{Data extraction and risk of bias assessment}

The relevant data from the studies were extracted independently by 2 authors (SI and $\mathrm{AH}$ ) using a pre-designed data extraction form and were checked for accuracy and completeness by a third reviewer (AA).

Two authors subsequently met to discuss their findings to resolve any discrepancies among the extracted data; any persistent discrepancies were resolved by consultation with a third author (AA).

The extracted data included publication characteristics (e.g., name of author, year, study design, and country), inclusion and exclusion criteria, sample size, population characteristics (e.g., patients' age and sex), intervention details (dose of drugs, route of administration, and period of treatment), outcomes, and design (length of follow-up, randomization, blinding, and allocation concealment). To obtain an ITT estimate, conservatively assuming that subjects lost to follow-up did not change their baseline consumption levels, the total number of patients in each group in baseline and the number of patients who met the response criteria were recorded.

The quality of the included studies was assessed by 2 authors (SI and HA) independently. Discrepancies were resolved in consultation with the third reviewer (AA). We used the Cochrane risk of bias tool to assess the quality of studies. This tool includes 7 specific domains: random sequence generation, allocation concealment, blinding of participants and personnel, blinding of outcome assessment, incomplete outcome data, selective reporting, and other sources of bias [41]. Studies were classified as having a low, high, or unclear risk of bias. Any discrepancies between reviewers were resolved in consultation with the third author (AA) until a consensus was reached.

\section{Data analysis}

Efficacy analyses were based on the reported numbers of confirmed cases of CL in each study and were performed for outcome measures if at least 2 studies could be pooled together.

We presented summary estimates from efficacy analyses primarily as relative risk (RR) to assess the strength of the effect; however, as an indicator of clinical significance, we also reported the risk difference (RD). All are reported with $95 \%$ confidence intervals (CIs). The meta-analyses were performed using a fixed-effects or random-effects method with inverse-variance weights and the DerSimonian-Laird estimator, respectively.

We assessed and quantified heterogeneity among studies using the chi-square test, with significance set at $\mathrm{p}$-value $<0.05$, and the $\mathrm{I}^{2}$ index, which is the percentage of the variation in the effect size estimation attributed to heterogeneity [42].

If heterogeneity was found to be present, meta-regression analyses were performed to identify possible relationships between efficacy and factors such as the sex ratio, sample size, study quality, and country location (New World or Old World).

A sensitivity analysis was used to verify the reliability of the results. A post hoc influence or sensitivity analysis was conducted by leaving out 1 study from the meta-analysis at a time and checking the consistency of the combined effect estimate.

Publication bias was assessed through visual inspection of the asymmetry of funnel plots. The Egger method, which is based on the asymmetry of funnel plots, was used to assess the influence of publication bias and, when significant evidence of publication bias was found, the trim-and-fill method was used to test and adjust for publication bias. The meta-analyses were performed using Stata version 12.0 (StataCorp., College Station, TX, USA).

\section{RESULTS}

The flow diagram presented in Figure 1 summarizes the study selection process.

A total of 1,570 reports were identified from the databases and by manual searching. After duplicates were removed, 1,034 papers remained, and after screening titles and abstracts, 57 studies were selected as potentially eligible to be included. After referring to the full texts, we ultimately included 10 studies (involving 1,006 participants; 550 in the miltefosine group and 456 in the MA group) in the efficacy meta-analyses. The characteristics of the included studies are presented in Table 1.

The studies were published between the years 2007 and 2014 (median, 2010). The mixture of geographic locations of studies was not very broad; 3 studies were conducted in Iran, 2 in Colombia, 2 in Brazil, 2 in Bolivia, and 1 in Pakistan. Except for 1 study [11] that was non-randomized, all studies were randomized parallel-group trials, and majority of the studies were not blinded. Most studies used an individual randomization design, but 3 used block randomization $[12,33,36]$. Seven studies had a mix of males and females [11,28,33,35,36,38,39], 1 study had only males [12], and in 2 studies the sex ratio was unclear [34,37]. The sample size ranged from a minimum of 30 to a maximum of 288 . Most studies recruited patients aged $>12$, while 1 RCT-enrolled only children (age < 12) [36]. Regarding parasite species, the majority of studies included L. braziliensis. One study provided a topical ointment intervention [39], whereas all the other studies provided oral miltefosine and injected MA. Oral miltefosine was compared in 7 studies with intramuscular injections of MA [11,12,33,34,36-38] and in 2 studies with intravenous MA [28,35]. The follow-up of the included studies ranged from 1 month to 6 months.

The risk-of-bias evaluations of the included studies are presented in Table 2. Most of the included studies were judged to have an unclear risk of selection bias, since the reporting of methods for randomization and allocation concealment was limited. Regarding the risk of performance bias due to non-blinding of patients, personnel, or outcome assessors, most of the included studies were judged at high risk for selection bias. Most studies were judged to be at low risk for attrition bias. Overall, studies were classified as having a high, low, or unclear risk of bias according to all components. 


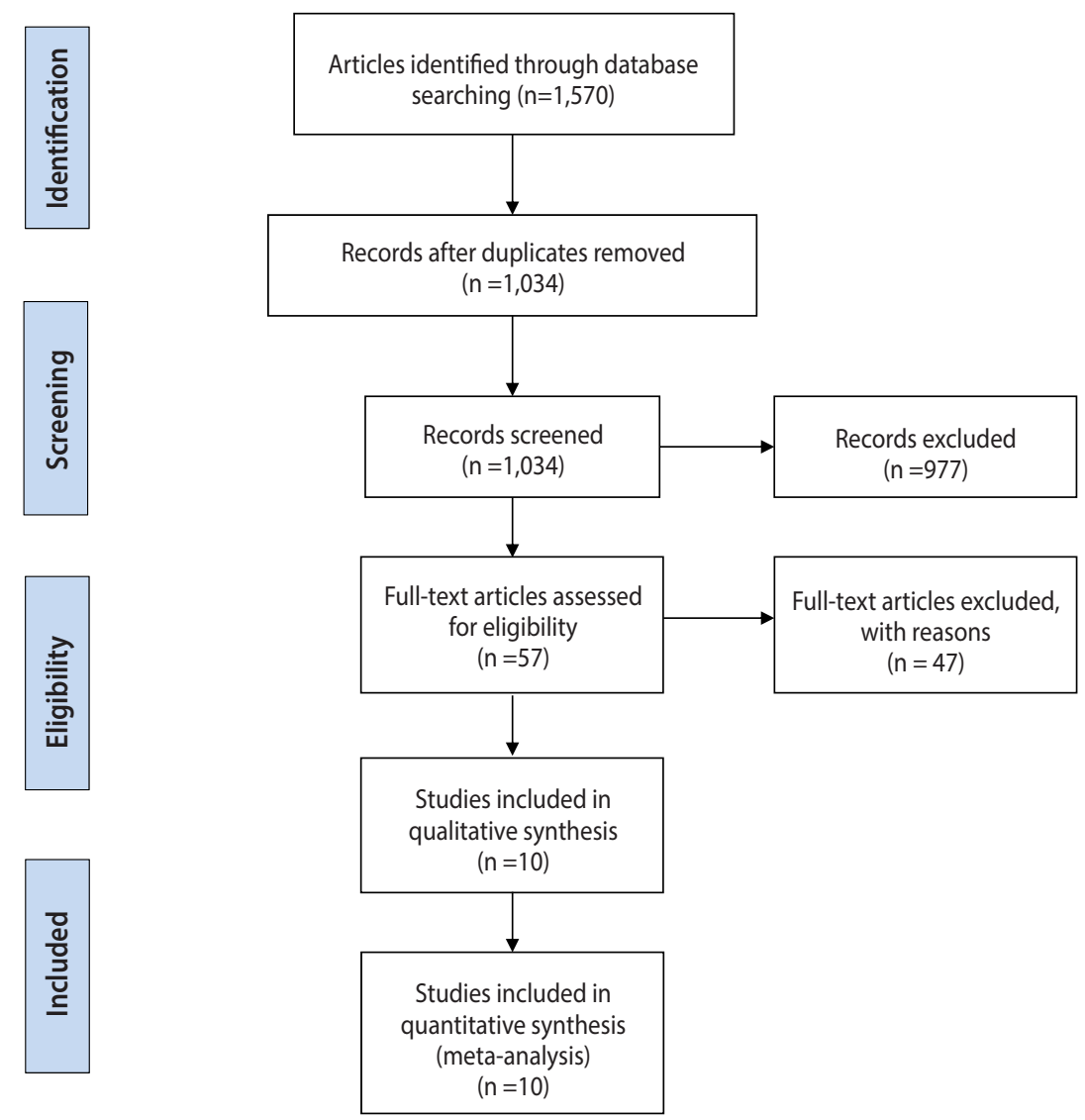

Figure 1. PRISMA (Preferred Reporting Items for System reviews and Meta-Analyses) flow diagram systematic search and review process.

In 1 study that did not report frequencies for the outcome of interest, the frequency was calculated based on the percentages reported in the article [34].

The included studies reported results for different follow-up periods.

Except for the 6-month post-treatment follow-up, for which both fixed-effects and random-effects methods were used for the meta-analyses, we used the random-effects method for all other post-treatment follow-up periods.

The results of the analyses of different follow-up periods are presented in Table 3.

In 1 of the 6 studies reporting the end-of-treatment cure rate and the 4 studies reporting the results at a 1-month post-treatment follow-up, the intervention was an ointment [39]; when excluding this study, the result did not change at either time point. In a metaanalysis of the 5 studies $[11,12,33,36,38]$ that reported results at a 3-month post-treatment follow-up, there was no significant difference between the efficacy of miltefosine and that of MA. One of the 5 studies was conducted in a special population (the Colombian Army) different in some characteristics, such as age range, sex (only male), and activity. In a sensitivity analysis excluding that study, which received the highest weight [12], miltefosine was significantly superior to MA in the complete cure rate at 3 months.
There were 8 studies $[11,12,28,33-36,38]$ that reported results from a 6-month post-therapy follow-up, which was the main focus of the literature and of this study. In a meta-analysis of these 8 studies $[11,12,28,33-36,38]$ with a fixed-effects and random-effects models, there was no significant difference between the efficacy of miltefosine and that of MA (Figure 2). However, in a sensitivity analysis excluding the study [12] with the highest weight (37.5\%), miltefosine was significantly superior to MA (Figure 3A).

Furthermore, using a random-effects model in the sensitivity analysis and excluding the study [12] with the highest weight (17.2\%), miltefosine was as effective as MA in the complete cure rate at 6 months (Figure 3B).

The results of analyses regarding parasite species are presented in Table 4.

There was no significant difference between the efficacy of miltefosine and that of MA with a random-effects model according to parasite species when the studies with a 6-month follow-up period were pooled.

There was no significant difference among any of the 4 studies with $L$. tropica/major, L. panamensis, L. major, and L. guyanensis species. Likewise, when the 4 studies with $L$. braziliensis were pooled $[12,28,34,38]$, there was no significant difference between the efficacy of miltefosine and that of MA. However, in a subgroup anal- 


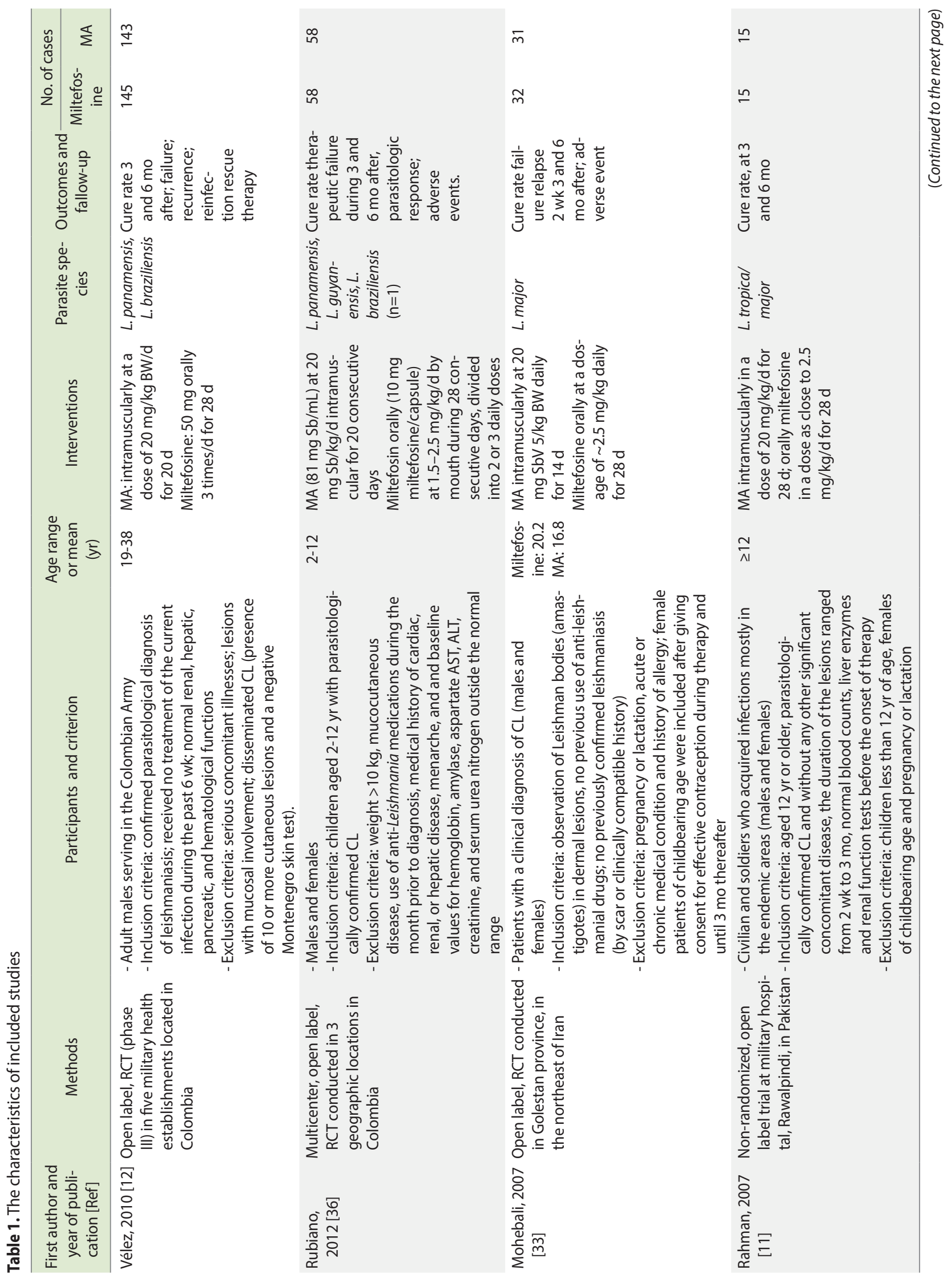




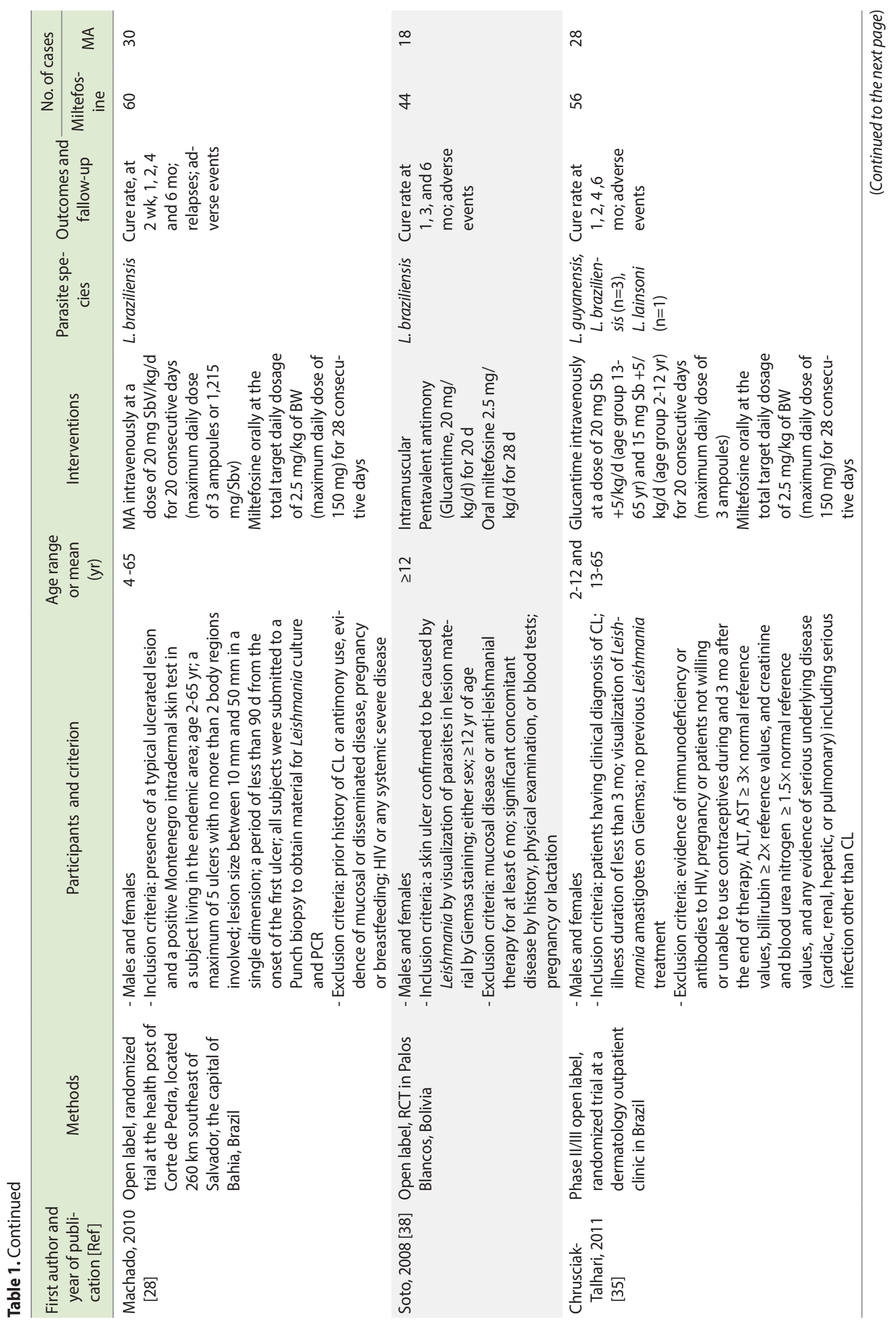




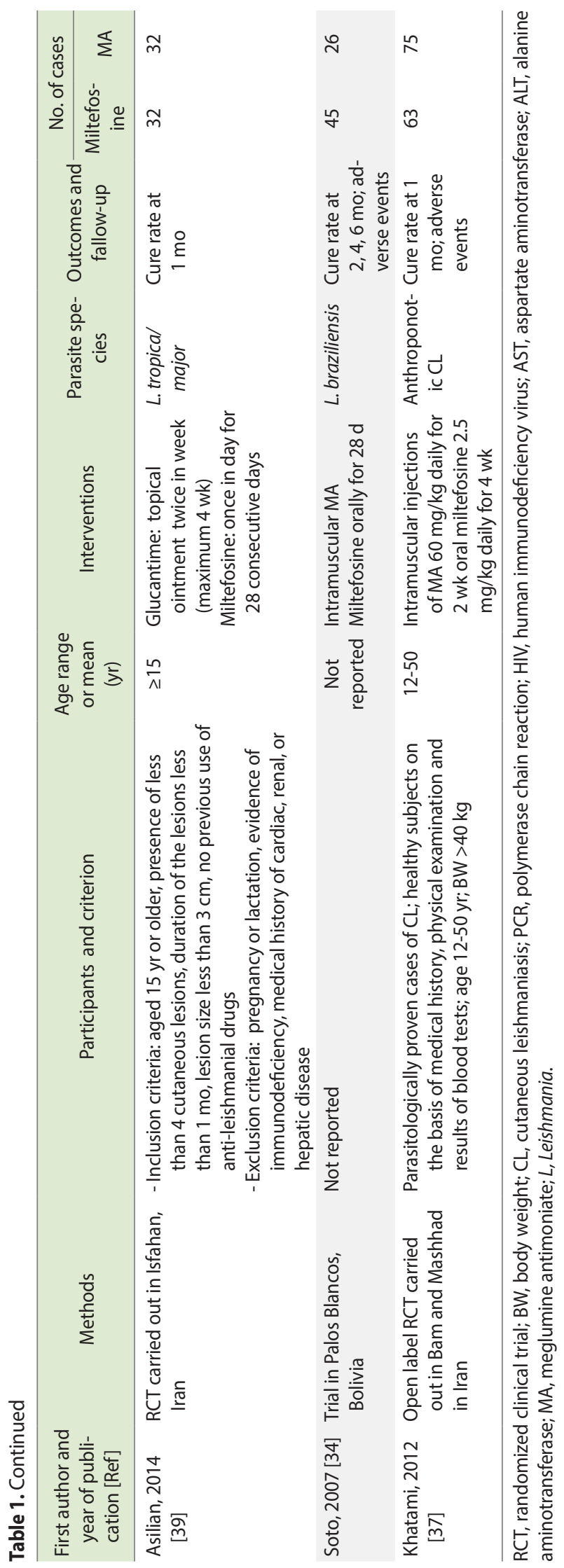

ysis excluding the studies with L. braziliensis, with the other 4 studies pooled using a fixed-effects model, miltefosine was significantly superior to MA (ITT: RR, 1.18 ; $95 \% \mathrm{CI}, 1.02$ to 1.37 ; RD, 0.12; $95 \%$ CI, 0.02 to $0.22: 293$ participants) [11,33,35,36], and the superiority of miltefosine persisted when a random-effects model was used (ITT: RR,1.15; 95\% CI, 1.01 to 1.32 ; RD, 0.11 ; 95\% CI, 0.01 to $0.21: 293$ participants).

Because the $\mathrm{I}^{2}$ statistic was found to be $56.8 \%$ (chi-square $=16.20$; $\mathrm{df}=7 ; \mathrm{p}=0.023$ ) in our review, a meta-regression analysis was conducted to investigate potential sources of heterogeneity, including the sex ratio, sample size, study quality, country type (New World or Old World), and MA injection type. Table 5 shows the results of the meta-regression between $\log (\mathrm{RR})$ and potential variables. As shown in Table 5, the results of the metaregression were not statistically significant, except for MA injection type, at the $\mathrm{p}=0.1$ level.

Publication bias was assessed using funnel plots (Supplementary Material 2), which found asymmetry. The Egger test $(t=3.35$; $\mathrm{p}=0.015$ ) found statistically significant publication bias (Supplementary Material 3). The trim-and-fill analysis revealed that 3 studies had been missed or trimmed (Supplementary Material 4). The trim-and-fill analysis demonstrated that including the missing studies did not change the results, and there was no significant difference between groups in either a fixed-effects model (ITT: RR, 0.96 ; $95 \%$ CI, 0.88 to 1.04 : 11 studies) or a random-effects model (ITT: RR, 0.97; 95\% CI, 0.82 to 1.12: 11 studies).

\section{DISCUSSION}

In this study, a PRISMA-compliant systematic review and meta-analysis was conducted based on clinical trials comparing miltefosine and MA in order to evaluate their efficacy in treating CL. In this meta-analysis, 10 clinical trials with 1,006 participants were included, exclusively comprising studies comparing miltefosine and MA as single-drug interventions. We observed significant variation in follow-up times. The 8 studies with 6-month follow-ups were the main focus of this study [11,12,28,33-36,38]. Using a random-effects model on an ITT basis, in the meta-analysis of 8 studies comparing miltefosine with MA for CL, we found no significant difference in the cure rate at 6 months. Using a random-effects model, the meta-analysis yielded an overall RR of 1.05 (95\% CI, 0.91 to 1.20 ) and an RD of 0.04 (95\% CI, -0.05 to 0.15 ; $\left.\mathrm{I}^{2}=60 \%\right)$. Visual inspection of the forest plot indicated significant heterogeneity between the study with the largest sample size [12] and the remaining studies. The study with the largest sample size was conducted on adult males serving in the Colombian Army, and it was the primary contributor to the high heterogeneity observed among the included studies; when this study was removed, the heterogeneity among the different studies decreased significantly. Differences in the sample size, age range, sex ratio, and other factors between that study and other studies might have contributed to the high heterogeneity. However, the main effect remained the same in the random-effects model, but changed in 


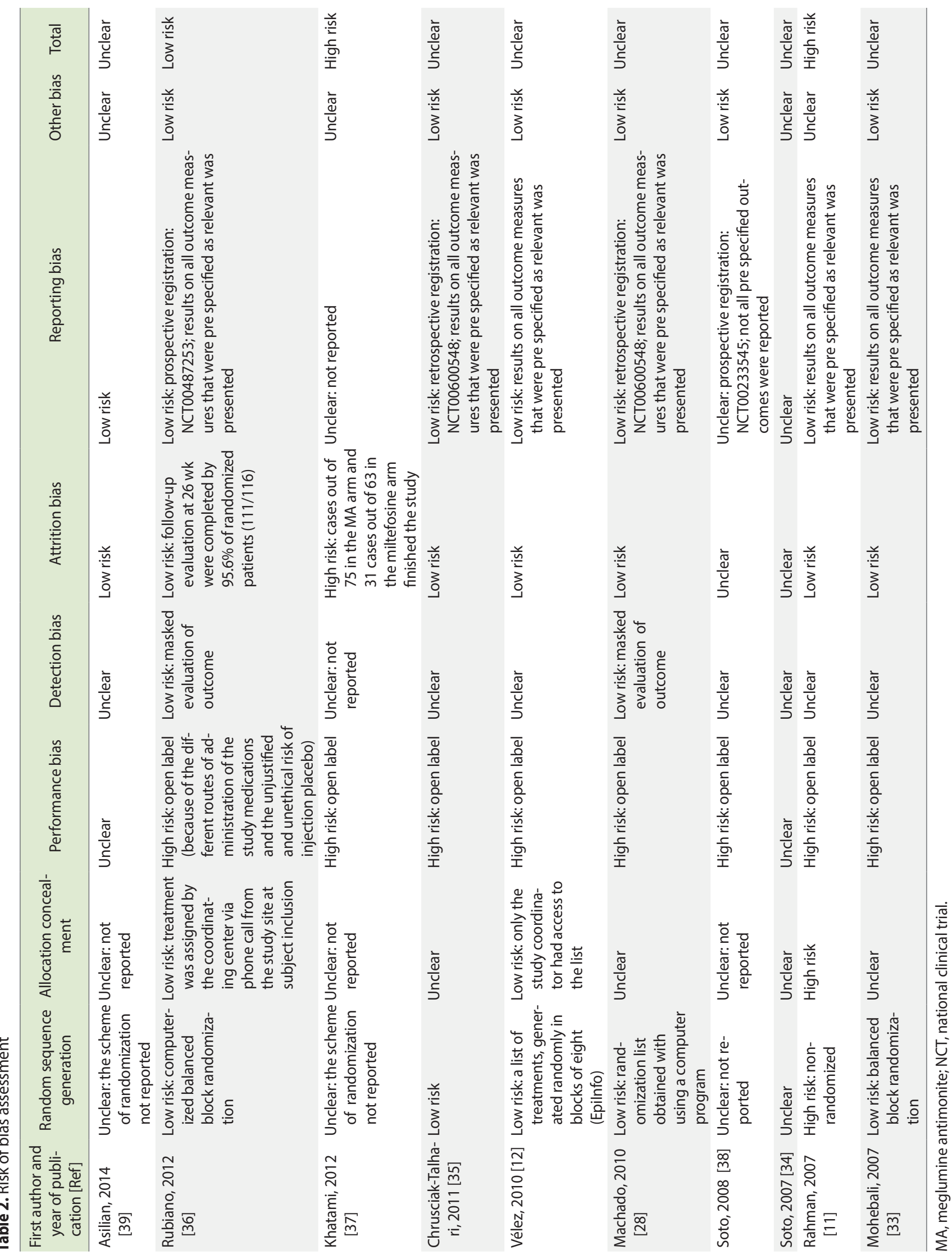


Table 3. Results of meta-analyses (comparison of the efficacy of miltefisine and meglumine antimoniate)

\begin{tabular}{|c|c|c|}
\hline Follow up period after end of treatment (d) [Ref] & $\begin{array}{l}\mathrm{ITT}(\mathrm{RR}, \mathrm{RD})[95 \% \mathrm{Cl}] \\
\text { (heterogeneity, \%) }\end{array}$ & $\begin{array}{l}\text { Effect measure (RR, RD) }[95 \% \mathrm{Cl}] \\
\text { (heterogeneity, \%) after excluding }\end{array}$ \\
\hline End of treatment $\left[11,28,35,36,38,39^{2}\right]$ & $\begin{array}{l}\text { RR: } 1.25(0.83,1.78) ; n=448 ; I^{2}=64 \\
\text { RD: } 0.08(-0.04,0.20) ; n=448 ; I^{2}=71\end{array}$ & $\begin{array}{l}\text { RR: } 1.19(0.74,1.93) ; n=384 ; I^{2}=67 \\
\text { RD: } 0.06(-0.06,0.20) ; n=384 ; I^{2}=72\end{array}$ \\
\hline $14[33]$ & $\begin{array}{l}\text { RR: } 1.10(0.80,1.51) ; n=63 \\
\text { RD: } 0.07(-0.15,0.29) ; n=63\end{array}$ & \\
\hline $30\left[35,37-39^{2}\right]$ & $\begin{array}{l}\text { RR: } 1.23(0.76,1.98) ; n=348 ; l^{2}=86 \\
\text { RD: } 0.07(-0.14,0.30) ; n=348 ; l^{2}=84\end{array}$ & $\begin{array}{l}\text { RR: } 0.98(0.69,1.39) ; n=284 ; I^{2}=68 \\
\text { RD: }-0.06(0.19,0.14) ; n=284 ; I^{2}=65\end{array}$ \\
\hline $60[28,34,35]$ & $\begin{array}{l}\text { RR: } 1.26(1.05,1.50) ; n=245 ; I^{2}=84 \\
\text { RD: } 0.16(0.04,0.28) ; n=245 ; I^{2}=0\end{array}$ & \\
\hline $90\left[11,12^{2}, 33,36,38\right]$ & $\begin{array}{l}\text { RR: } 1.03(0.85,1.24) ; n=559 ; l^{2}=75 \\
\text { RD: } 0.03(-0.12,0.18) ; n=559 ; l^{2}=77\end{array}$ & $\begin{array}{l}\text { RR: } 1.12(1.00,1.27) ; n=271 ; I^{2}=11 \\
\text { RD: } 0.10(0.01,0.20) ; n=271 ; I^{2}=14\end{array}$ \\
\hline $120[34,35]$ & $\begin{array}{l}\text { RR: } 1.07(0.84,1.36) ; n=155 ; I^{2}=39 \\
\text { RD: } 0.05(-0.08,0.19) ; n=155 ; I^{2}=18\end{array}$ & \\
\hline $180^{1}\left[11,12^{2}, 28,33-36,38\right]$ & $\begin{array}{l}\text { RR: } 1.01(0.92,1.11) ; n=804 ; l^{2}=39 \\
\text { RD: } 0.01(-0.05,0.07) ; n=804 ; l^{2}=60\end{array}$ & $\begin{array}{l}\text { RR: } 1.14(1.03,1.27) ; n=516 ; I^{2}=35 \\
\text { RD: } 0.01(-0.05,0.07) ; n=516 ; I^{2}=60\end{array}$ \\
\hline $180\left[11,12^{2}, 28,33-36,38\right]$ & $\begin{array}{l}\text { RR: } 1.05(0.91,1.20) ; n=804 ; l^{2}=56 \\
\text { RD: } 0.04(-0.05,0.15) ; n=804 ; l^{2}=60\end{array}$ & $\begin{array}{l}\text { RR: } 1.10(0.97,1.25) ; n=516 ; I^{2}=35 \\
\text { RD: } 0.08(-0.01,0.16) ; n=516 ; I^{2}=26\end{array}$ \\
\hline
\end{tabular}

$\mathrm{ITT}$, intention to treat; $\mathrm{RR}$, relative risk; $\mathrm{RD}$, risk difference; $\mathrm{Cl}$, confidence interval.

'Fixed method.

${ }^{2}$ Excluded studies.

Table 4. Comparison of the efficacy of miltefisine and meglumine antimoniate regarding to parasite species

\begin{tabular}{|c|c|}
\hline Parasite species [Ref] & ITT (RR, RD) [95\% Cl] (heterogeneity, \%) \\
\hline L. tropica/major, L. panamensis/major, L. guyanensis, L. braziliensis $[11,12,28,33-36,38]^{1}$ & $\begin{array}{l}\text { RR: } 1.14(1.03,1.27) ; n=804 ; l^{2}=35 \\
\text { RD: } 0.01(-0.05,0.07) ; n=804 ; l^{2}=60\end{array}$ \\
\hline L. tropica/major, L. panamensis/major, L. guyanensis, L. braziliensis $[11,12,28,33-36,38]$ & $\begin{array}{l}\text { RR: } 1.05(0.91,1.20) ; n=804 ; l^{2}=56 \\
\text { RD: } 0.04(-0.05,0.15) ; n=804 ; l^{2}=60\end{array}$ \\
\hline L. tropica/major [11] & $\begin{array}{l}\text { RR: } 1.30(0.86,1.95) ; n=30 \\
\text { RD: } 0.20(-0.09,0.49) ; n=30\end{array}$ \\
\hline L. panamensis [36] & $\begin{array}{l}\text { RR: } 1.20(0.97,1.47) ; n=116 \\
R D: 0.13(-0.01,0.29) ; n=166\end{array}$ \\
\hline L. major [33] & $\begin{array}{l}\text { RR: } 1.01(0.79,1.28) ; n=63 \\
\text { RD: }-0.01(-0.18,0.20) ; n=63\end{array}$ \\
\hline L. guyanensis [35] & $\begin{array}{l}\text { RR: } 1.33(0.90,1.95) ; n=84 \\
\text { RD: } 0.17(-0.04,0.39) ; n=84\end{array}$ \\
\hline L. braziliensis $[12,28,34,38]$ & $\begin{array}{l}\text { RR: } 0.92(0.82,1.04) ; n=511 ; l^{2}=0 \\
\text { RD: }-0.05(-0.13,0.02) ; n=511 ; I^{2}=0\end{array}$ \\
\hline L. tropica/major, L. panamensis/major, L. guyanensis $[11,33,35,36]^{1}$ & $\begin{array}{l}\text { RR: } 1.18(1.02,1.37) ; n=293 ; l^{2}=0 \\
\text { RD: } 0.12(0.02,0.22) ; n=293 ; l^{2}=0\end{array}$ \\
\hline L. tropica/major, L. panamensis/major, L. guyanensis $[11,33,35,36]$ & $\begin{array}{l}\text { RR: } 1.15(1.01,1.32) ; n=293 ; I^{2}=0 \\
\text { RD: } 0.11(0.01,0.21) ; n=293 ;\left.\right|^{2}=0\end{array}$ \\
\hline
\end{tabular}

$\mathrm{ITT}$, intention to treat; $\mathrm{RR}$, relative risk; $\mathrm{RD}$, risk difference; $\mathrm{Cl}$, confidence interval; L, Leishmania. ${ }^{1}$ Fixed method.

the fixed-effects model to favor miltefosine.

Some evidence suggested that the species of parasite is a factor influencing the cure rate of drugs and that the differences in the therapeutic response between the studies carried out in different regions may be partially attributed to the Leishmania species [26,30]. However, no clear consensus emerged regarding the Leishmania species.

Interestingly, in our study, in all the included studies with spe- cies other than L. braziliensis, miltefosine was superior, with no significant difference in the cure rate at 6 months. Although in subgroup analyses based on parasite species, no significant differences were detected in any of the subgroups in the cure rate at 6 months, when pooling the 4 studies with species other than $L$. braziliensis (including L. panamensis, L. guyanensis, L. tropica, and L. major) to increase the power, there was a significant difference favoring miltefosine in the cure rate at 6 months with no heteroge- 


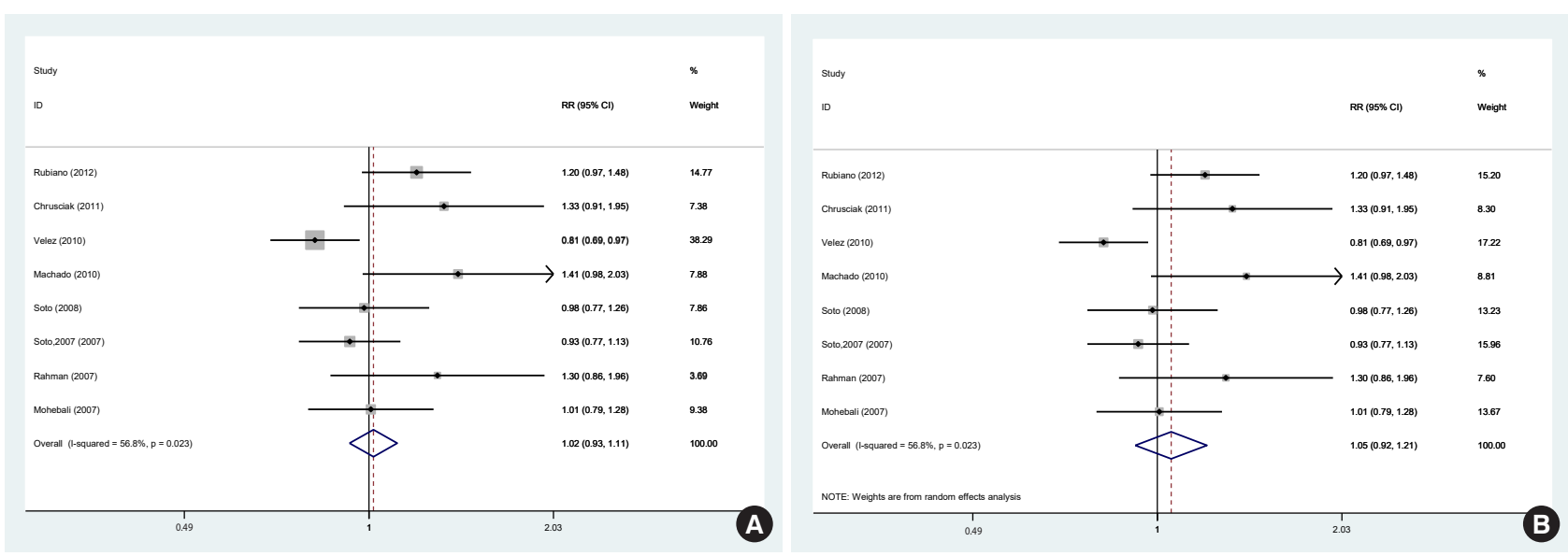

Figure 2. Meta-analysis of the eight studies evaluating miltefosine compared to meglumine antimoniate in the rate of complete cure at 6 months of follow up (A) fixed-effects model, (B) random-effects model. $\mathrm{RR}$, relative risk; $\mathrm{Cl}$, confidence interval.

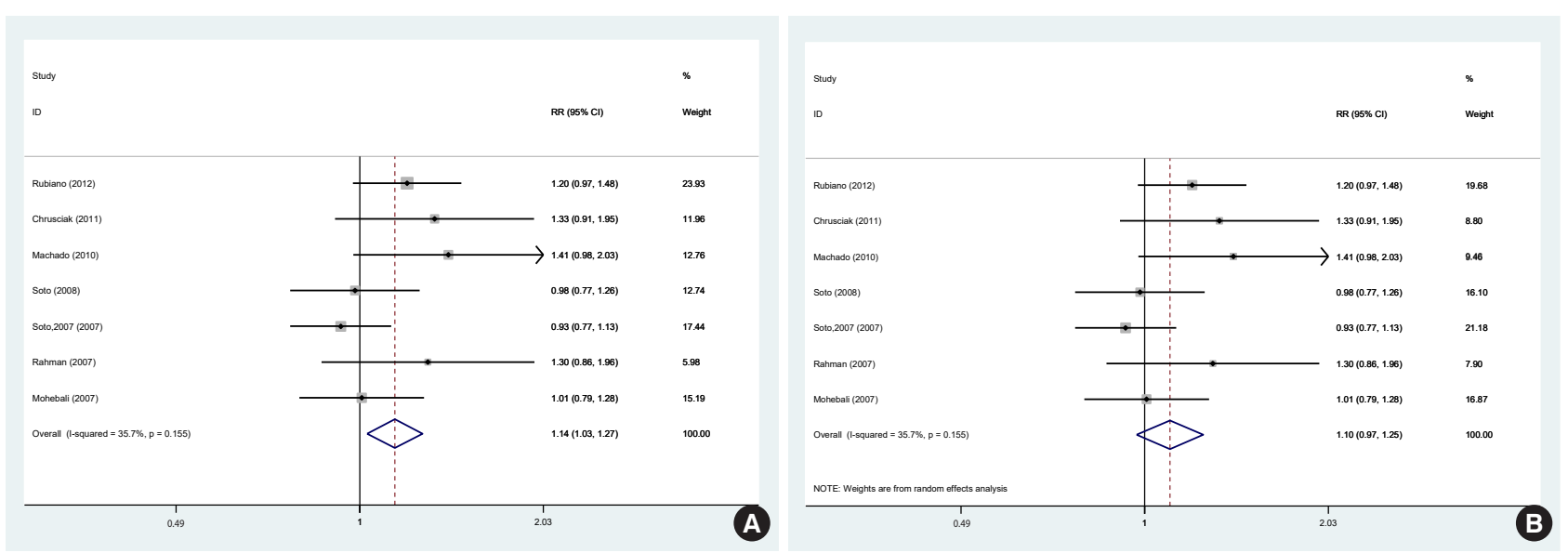

Figure 3. Meta-analysis of the seven studies evaluating miltefosine compared to meglumine antimoniate in the rate of complete cure at 6 months of follow up (A) fixed-effects model, (B) random-effects model. $\mathrm{RR}$, relative risk; $\mathrm{Cl}$, confidence interval.

Table 5. The results of meta-regression

\begin{tabular}{|c|c|c|c|c|c|c|c|}
\hline Factors & Level & Coefficient & $t$-value & $p$-value & I-squared (\%) & tau2 & $\begin{array}{l}\text { Adjusted R- } \\
\text { squared (\%) }\end{array}$ \\
\hline Country category & & 0.04 & 0.25 & 0.81 & 10 & 0.03 & -25.13 \\
\hline Sex ratio & & 0.29 & 0.56 & 0.59 & 14 & 0.03 & -25.15 \\
\hline Sample size & & -0.00 & -1.50 & 0.18 & 6 & 0.02 & 25.41 \\
\hline Injection type & & 0.03 & 2.19 & 0.07 & 13 & 0.01 & 53.98 \\
\hline \multirow[t]{3}{*}{ Quality of study } & Low & Reference & - & - & 3 & 0.02 & 1.16 \\
\hline & Unclear & -0.17 & -0.77 & 0.47 & & & \\
\hline & High & 0.10 & 0.29 & 0.78 & & & \\
\hline
\end{tabular}

neity. When pooling studies with $L$. braziliensis [12,28,34,38], MA was superior with no significant difference in the cure rate at 6 months with zero heterogeneity. Therefore, the subgroup analyses conducted to evaluate species-specific efficacy suggest that miltefosine may be superior to MA in species other than L. braziliensis and non-inferior in L. braziliensis. This demonstrates that the ef- fect of drugs likely depends on the parasite species.

We found similar results for the 4 studies with a 3-month follow up period. After excluding 1 study that included L. braziliensis, and pooling the remaining 3 studies with other species, miltefosine was significantly superior to MA.

These findings are similar, to some extent, to the results of a 
study conducted in Brazil comparing the therapeutic response of CL due to L. braziliensis and L. guyanensis to MA, which showed that Leishmania species was an important factor in predicting the outcome of CL treated with MA; in particular, the failure rate was higher in patients infected with L. guyanensiss [26].

The findings of the present study are to some extent inconsistent with previous systematic reviews and meta-analyses [40]. The conflicting results between our meta-analyses and previous metaanalyses may be due to the inclusion of updated studies from the Old World that were not included in previous meta-analyses. It is important to bear in mind that the majority of the studies in previous meta-analyses included L. braziliensis, whereas the updated studies in our review mainly analyzed other species. Nonetheless, in subgroup analyses we found that miltefosine was non-inferior to MA in L. braziliensis, similarly to previous meta-analyses, and superior in species other than L. braziliensis.

Meta-regressions were conducted for the sex ratio, sample size, study quality, and country type (New World or Old World). Among these characteristics, only the route of administration of MA was significant. Performing meta-regressions adjusted for confounding variables was limited by the relatively small number of studies.

\section{Publication bias}

The analysis of publication bias was limited by the relatively small number of studies. With this limitation in mind, publication bias was assessed through a funnel plot, the Egger test, and a trim-and-fill analysis. The funnel plot and Egger test showed the presence of publication bias in the included studies. However, the trim-and-fill analysis demonstrated that the impact of publication bias was within an acceptable range, and the inclusion of the missing studies did not change the overall conclusions.

Overall, the findings of our study show that although the cure rate of miltefosine treatment compared with MA varied among Leishmania species, miltefosine was at least as good as MA in treating CL caused by L. braziliensis and superior in treating leishmaniasis caused by other species. In terms of the most important advantage of miltefosine (its comfort for patients) [33], the practical importance of this finding is that miltefosine can be administered for $\mathrm{CL}$ as an oral agent that will be approximately as effective as standard injections of MA.

Some mild adverse gastrointestinal effects such as anorexia, nausea, abdominal pain, diarrhea, and vomiting are common and were reported with miltefosine [29-31]. However, they do not usually require suspension of treatment [12]. Therefore, miltefosine could be a safe and effective alternative to MA and could be especially helpful in regions where parasites are resistant to MA.

One major problem with miltefosine is teratogenicity in reproductive-age female. Using contraception during therapy and for 4-5 months after treatment completion is necessary due to the long half-life of miltefosine [43].

The major limitation of our study was its relatively small sample size and the paucity of relevant studies, which restricted the assessment of heterogeneity and publication bias. Neither alloca- tion concealment nor blinding was performed in the majority of the included studies.

\section{CONCLUSION}

This meta-analysis found that miltefosine seems to be more effective than MA in treating CL, at least in species other than $L$. braziliensis.

\section{SUPPLEMENTARY MATERIALS}

Supplementary materials are available at http://www.e-epih.org/.

\section{CONFLICT OF INTEREST}

The authors have no conflicts of interest to declare for this study.

\section{ACKNOWLEDGEMENTS}

None.

\section{ORCID}

Sohrab Iranpour: https://orcid.org/0000-0002-0802-087X; Ali Hosseinzadeh: http://orcid.org/0000-0002-0055-9408; Abbas Alipour: https://orcid.org/0000-0003-0781-3728

\section{REFERENCES}

1. Reithinger R, Dujardin JC, Louzir H, Pirmez C, Alexander B, Brooker S. Cutaneous leishmaniasis. Lancet Infect Dis 2007;7:581596.

2. Alvar J, Vélez ID, Bern C, Herrero M, Desjeux P, Cano J, et al. Leishmaniasis worldwide and global estimates of its incidence. PLoS One 2012;7:e35671.

3. World Health Organizaion. Leishmaniasis [cited 2014 Dec 3]. Available from: http://www.who.int/mediacentre/factsheets/ fs375/en/.

4. Khan SJ, Muneeb S. Cutaneous leishmaniasis in Pakistan. Dermatol Online J 2005;11:4.

5. Rahman SB, Bari AU. Laboratory profile in patients of cutaneous leishmaniasis from various regions of Pakistan. J Coll Physicians Surg Pak 2003;13:313-316.

6. Mujtaba G, Khalid M. Cutaneous leishmaniasis in Multan, Pakistan. Int J Dermatol 1998;37:843-845.

7. Jones TC, Johnson WD Jr, Barretto AC, Lago E, Badaro R, Cerf B, et al. Epidemiology of American cutaneous leishmaniasis due to Leishmania braziliensis brasiliensis. J Infect Dis 1987;156:73-83.

8. Miranda Lessa M, Andrade Lessa H, Castro TW, Oliveira A, Scherifer A, Machado P, et al. Mucosal leishmaniasis: epidemiological and clinical aspects. Braz J Otorhinolaryngol 2007;73:843-847.

9. Turetz ML, Machado PR, Ko AI, Alves F, Bittencourt A, Almeida $\mathrm{RP}$, et al. Disseminated leishmaniasis: a new and emerging form 
of leishmaniasis observed in northeastern Brazil. J Infect Dis 2002; 186:1829-1834

10. Soto J, Soto P. Current situation and future of antileishmanial therapy in Colombia. Biomedica 2006;26 Suppl 1:194-206 (Spanish).

11. Rahman SB, ul Bari A, Mumtaz N. Miltefosine in cutaneous leishmaniasis. J Coll Physicians Surg Pak 2007;17:132-135.

12. Vélez I, López L, Sánchez X, Mestra L, Rojas C, Rodríguez E. Efficacy of miltefosine for the treatment of American cutaneous leishmaniasis. Am J Trop Med Hyg 2010;83:351-356.

13. Salam N, Al-Shaqha WM, Azzi A. Leishmaniasis in the Middle East: incidence and epidemiology. PLoS Negl Trop Dis 2014;8:e3208.

14. Herwaldt BL, Berman JD. Recommendations for treating leishmaniasis with sodium stibogluconate (Pentostam) and review of pertinent clinical studies. Am J Trop Med Hyg 1992;46:296-306.

15. Croft SL, Sundar S, Fairlamb AH. Drug resistance in leishmaniasis. Clin Microbiol Rev 2006;19:111-126.

16. Croft SL, Seifert K, Yardley V. Current scenario of drug development for leishmaniasis. Indian J Med Res 2006;123:399-410.

17. González U, Pinart M, Rengifo-Pardo M, Macaya A, Alvar J, Tweed JA. Interventions for American cutaneous and mucocutaneous leishmaniasis. Cochrane Database Syst Rev 2009:CD004834.

18. Santos JB, de Jesus AR, Machado PR, Magalhães A, Salgado K, Carvalho EM, et al. Antimony plus recombinant human granulocyte-macrophage colony-stimulating factor applied topically in low doses enhances healing of cutaneous leishmaniasis ulcers: a randomized, double-blind, placebo-controlled study. J Infect Dis 2004;190:1793-1796.

19. Arevalo I, Tulliano G, Quispe A, Spaeth G, Matlashewski G, Llanos-Cuentas A, et al. Role of imiquimod and parenteral meglumine antimoniate in the initial treatment of cutaneous leishmaniasis. Clin Infect Dis 2007;44:1549-1554.

20. Kedzierski L, Sakthianandeswaren A, Curtis JM, Andrews PC, Junk PC, Kedzierska K. Leishmaniasis: current treatment and prospects for new drugs and vaccines. Curr Med Chem 2009;16: 599-614.

21. Ouellette M, Drummelsmith J, Papadopoulou B. Leishmaniasis: drugs in the clinic, resistance and new developments. Drug Resist Updat 2004;7:257-266.

22. Asilian A, Sadeghinia A, Faghihi G, Momeni A. Comparative study of the efficacy of combined cryotherapy and intralesional meglumine antimoniate (Glucantime ${ }^{\circledR}$ ) vs. cryotherapy and intralesional meglumine antimoniate (Glucantime ${ }^{\circledR}$ ) alone for the treatment of cutaneous leishmaniasis. Int J Dermatol 2004;43:281283.

23. Hadighi R, Mohebali M, Boucher P, Hajjaran H, Khamesipour A, Ouellette M. Unresponsiveness to Glucantime treatment in Iranian cutaneous leishmaniasis due to drug-resistant Leishmania tropica parasites. PLoS Med 2006;3:e162.

24. Alvar J, Croft S, Olliaro P. Chemotherapy in the treatment and control of leishmaniasis. Adv Parasitol 2006;61:223-274.

25. Berman JD. Development of miltefosine for the leishmaniases. Mini Rev Med Chem 2006;6:145-151.

26. Romero GA, Guerra MV, Paes MG, Macêdo VO. Comparison of cutaneous leishmaniasis due to Leishmania (Viannia) braziliensis and L. (V.) guyanensis in Brazil: therapeutic response to meglumine antimoniate. Am J Trop Med Hyg 2001;65:456-465.

27. Unger A, O’Neal S, Machado PR, Guimarães LH, Morgan DJ, Schriefer A, et al. Association of treatment of American cutaneous leishmaniasis prior to ulcer development with high rate of failure in northeastern Brazil. Am J Trop Med Hyg 2009;80:574579.

28. Machado PR, Ampuero J, Guimarães LH, Villasboas L, Rocha AT, Schriefer A, et al. Miltefosine in the treatment of cutaneous leishmaniasis caused by Leishmania braziliensis in Brazil: a randomized and controlled trial. PLoS Negl Trop Dis 2010;4:e912.

29. Sundar S, Jha TK, Thakur CP, Engel J, Sindermann H, Fischer C, et al. Oral miltefosine for Indian visceral leishmaniasis. N Engl J Med 2002;347:1739-1746.

30. Soto J, Arana BA, Toledo J, Rizzo N, Vega JC, Diaz A, et al. Miltefosine for new world cutaneous leishmaniasis. Clin Infect Dis 2004;38:1266-1272.

31. Soto J, Toledo J, Gutierrez P, Nicholls RS, Padilla J, Engel J, et al. Treatment of American cutaneous leishmaniasis with miltefosine, an oral agent. Clin Infect Dis 2001;33:E57-E61.

32. Van Thiel PP, Leenstra T, Kager PA, de Vries HJ, van Vugt M, van der Meide WF, et al. Miltefosine treatment of Leishmania major infection: an observational study involving Dutch military personnel returning from northern Afghanistan. Clin Infect Dis 2010; 50:80-83.

33. Mohebali M, Fotouhi A, Hooshmand B, Zarei Z, Akhoundi B, Rahnema A, et al. Comparison of miltefosine and meglumine antimoniate for the treatment of zoonotic cutaneous leishmaniasis (ZCL) by a randomized clinical trial in Iran. Acta Trop 2007;103: 33-40.

34. Soto J, Toledo JT. Oral miltefosine to treat new world cutaneous leishmaniasis. Lancet Infect Dis 2007;7:7.

35. Chrusciak-Talhari A, Dietze R, Chrusciak Talhari C, da Silva RM, Gadelha Yamashita EP, de Oliveira Penna G, et al. Randomized controlled clinical trial to access efficacy and safety of miltefosine in the treatment of cutaneous leishmaniasis caused by Leishmania (Viannia) guyanensis in Manaus, Brazil. Am J Trop Med Hyg 2011;84:255-260.

36. Rubiano LC, Miranda MC, Muvdi Arenas S, Montero LM, Rodríguez-Barraquer I, Garcerant D, et al. Noninferiority of miltefosine versus meglumine antimoniate for cutaneous leishmaniasis in children. J Infect Dis 2012;205:684-692.

37. Khatami A, Rahshenas M, Bahrami M, Ghoorchi MH, Eskandari SE, Sharifi I, et al. Miltefosine in treatment of cutaneous leishmaniasis: a randomized controlled trial: P-31. Br J Dermatol 2012; 167:e23.

38. Soto J, Rea J, Balderrama M, Toledo J, Soto P, Valda L, et al. Efficacy of miltefosine for Bolivian cutaneous leishmaniasis. Am J Trop Med Hyg 2008;78:210-211.

39. Asilian A, Omrani S, Nilforoushzadeh MA. Comparing the effects of topical miltefosine and glucantime in treatment of cutaneous leishmaniasis. J Isfahan Med Sch 2014;31:2257-2263 (Per- 
sian).

40. Reveiz L, Maia-Elkhoury AN, Nicholls RS, Romero GA, Yadon ZE. Interventions for American cutaneous and mucocutaneous leishmaniasis: a systematic review update. PLoS One 2013;8:e61843.

41. Higgins JP, Altman DG, Gøtzsche PC, Jüni P, Moher D, Oxman $\mathrm{AD}$, et al. The Cochrane Collaboration's tool for assessing risk of bias in randomised trials. BMJ 2011;343:d5928.
42. Higgins JP, Thompson SG. Quantifying heterogeneity in a metaanalysis. Stat Med 2002;21:1539-1558.

43. Dorlo TP, van Thiel PP, Huitema AD, Keizer RJ, de Vries HJ, Beijnen $\mathrm{JH}$, et al. Pharmacokinetics of miltefosine in Old World cutaneous leishmaniasis patients. Antimicrob Agents Chemother 2008;52:2855-2860. 